\title{
High repetition rate laser-induced breakdown spectroscopy using acousto-optically gated detection
}

\author{
PAVEL POŘÍZKA, BENJAMIN KLESSEN, JOZEF KAISER, \\ IGOR GORNUSHKIN, ULRICH PANNE, JENS RIEDEL
}

Citation: Review of Scientific Instruments 85, 073104 (2014)

ISSN: 0034-6748

DOI: http://dx.doi.org/10.1063/1.4890337

Final PDF version

(C) 2014 AIP Publishing LLC. This article may be downloaded for personal use only. Any other use requires prior permission of the author and AIP Publishing. The following article appeared in Review of Scientific Instruments 85, 073104 (2014); doi: 10.1063/1.4890337 and may be found at http://dx.doi.org/10.1063/1.4890337 


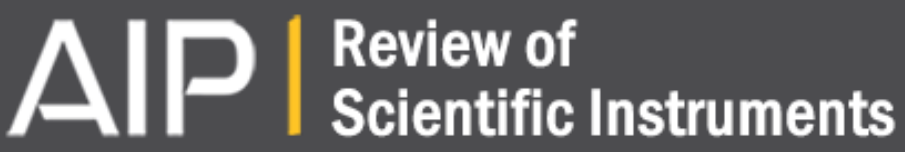

\section{High repetition rate laser-induced breakdown spectroscopy using acousto-optically gated detection}

Pavel Poízka, Benjamin Klessen, Jozef Kaiser, Igor Gornushkin, Ulrich Panne, and Jens Riedel

Citation: Review of Scientific Instruments 85, 073104 (2014); doi: 10.1063/1.4890337

View online: http://dx.doi.org/10.1063/1.4890337

View Table of Contents: http://scitation.aip.org/content/aip/journal/rsi/85/7?ver=pdfcov

Published by the AIP Publishing

\section{Articles you may be interested in}

High coherent bi-chromatic laser with gigahertz splitting produced by the high diffraction orders of acousto-optic modulator used for coherent population trapping experiments

Rev. Sci. Instrum. 82, 123104 (2011); 10.1063/1.3665986

Creation of arbitrary spectra with an acousto-optic modulator and an injection-locked diode laser

Rev. Sci. Instrum. 82, 083108 (2011); 10.1063/1.3626903

Toward quantitative deuterium analysis with laser-induced breakdown spectroscopy using atmospheric-pressure helium gas

J. Appl. Phys. 107, 023301 (2010); 10.1063/1.3282801

Discrimination of microbiological samples using femtosecond laser-induced breakdown spectroscopy Appl. Phys. Lett. 89, 163903 (2006); 10.1063/1.2361270

Simultaneous elemental analysis system using laser induced breakdown spectroscopy

Rev. Sci. Instrum. 72, 1625 (2001); 10.1063/1.1338486

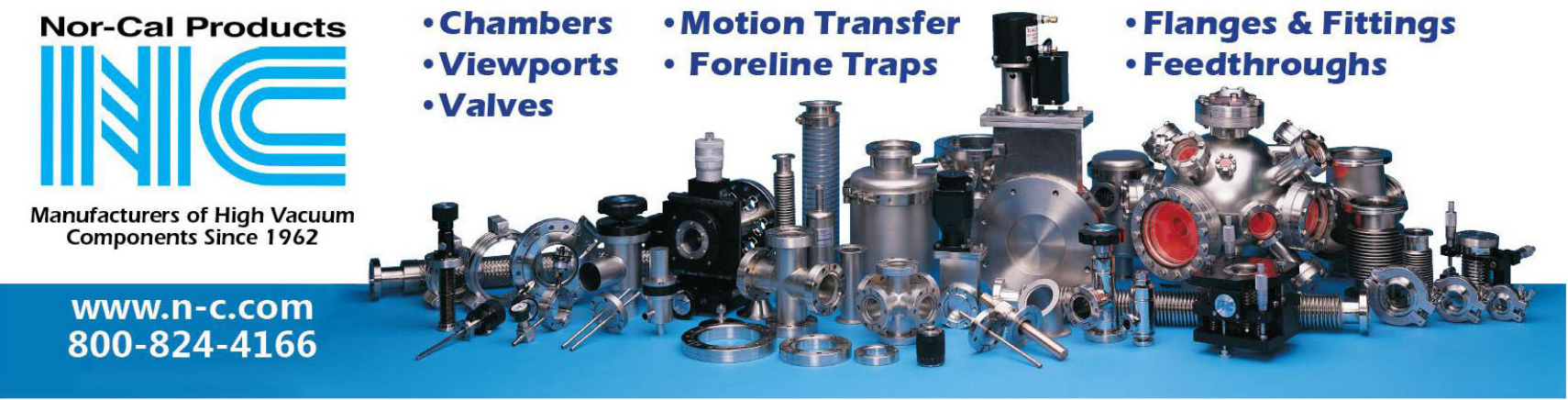




\title{
High repetition rate laser-induced breakdown spectroscopy using acousto-optically gated detection
}

\author{
Pavel Pořízka, ${ }^{1,2}$ Benjamin Klessen, ${ }^{1}$ Jozef Kaiser,${ }^{2}$ Igor Gornushkin, ${ }^{1}$ Ulrich Panne,,${ }^{1,3}$ and \\ Jens Riedel ${ }^{1}$ \\ ${ }^{1}$ BAM Federal Institute for Materials Research and Testing, Richard-Willstätter-Straße 11, D-12489 Berlin, \\ Germany \\ ${ }^{2}$ Institute of Physical Engineering, Faculty of Mechanical Engineering, Brno University of Technology, \\ Technická 2896/2, 61669 Brno, Czech Republic \\ ${ }^{3}$ Chemistry Department, Humboldt Universität zu Berlin, Brook-Taylor-Straße 2, D-12489 Berlin, Germany
}

(Received 19 November 2013; accepted 3 July 2014; published online 24 July 2014)

\begin{abstract}
This contribution introduces a new type of setup for fast sample analysis using laser-induced breakdown spectroscopy (LIBS). The novel design combines a high repetition rate laser (up to $50 \mathrm{kHz}$ ) as excitation source and an acousto-optical modulator (AOM) as a fast switch for temporally gating the detection of the emitted light. The plasma radiation is led through the active medium of the AOM where it is diffracted on the transient ultrasonic Bragg grid. The diffracted radiation is detected by a compact Czerny-Turner spectrometer equipped with a CCD line detector. Utilizing the new combination of high repetition rate lasers and AOM gated detection, rapid measurements with total integration times of only $10 \mathrm{~ms}$ resulted in a limit of detection (LOD) of $0.13 \mathrm{wt} . \%$ for magnesium in aluminum alloys. This short integration time corresponds to 100 analyses/s. Temporal gating of LIP radiation results in improved LODs and consecutively higher sensitivity of the LIBS setup. Therefore, an AOM could be beneficially utilized to temporally detect plasmas induced by high repetition rate lasers. The AOM in combination with miniaturized Czerny-Turner spectrometers equipped with CCD line detectors and small footprint diode pumped solid state lasers results in temporally gateable compact LIBS setups. @ 2014 AIP Publishing LLC. [http://dx.doi.org/10.1063/1.4890337]
\end{abstract}

\section{INTRODUCTION}

In recent years laser-induced breakdown spectroscopy (LIBS) has become an increasingly popular analytical method. Its major advantages are its multi-element capacity, experimental simplicity, and the lack of sample preparation. ${ }^{1-4}$ Briefly, a strong non-resonant laser pulse is used to ablate, atomize, excite, and ionize a small amount of the sample, thus forming transient microplasma. While relaxing, the matter inside the formed plasma plume emits light. In the first few hundred nanoseconds the plasma is typically very hot; its radiation mainly stems from electron-ion recombination and bremsstrahlung. The radiation is spectrally broad and contains no information on the elemental composition of the plasma. After this period, atoms and ions of the analyte undergo relaxation between well-defined energy states and emit light with characteristic wavelengths. These optical emission spectra are often very dense, since they contain spectrochemical information from all elements present in the ablated matter. To obtain maximum information, the emitted light should, therefore, be (a) temporally gated to discriminate against the early broadband emission and (b) detected with a sufficiently high spectral resolution to resolve contributions from all individual species involved.

LIBS has been successfully applied for quantitative analysis of trace elements in solids, ${ }^{5,6}$ liquids, ${ }^{7-9}$ and gases. ${ }^{10,11}$ Numerous experimental setups have been introduced with different laser sources and spectrometer types. ${ }^{12}$ Because no sample preparation is needed, LIBS is perfectly suited for sorting and mapping applications. ${ }^{13}$ Since the recorded plas- mas have lifetimes of only several $\mu \mathrm{s}$, subsequent spectra can be recorded within a short time interval; this makes LIBS also a perfect candidate for mapping 2D surfaces. Typical plasma lifetimes are $\sim 10 \mu \mathrm{s}$ so the duty cycle loosely dictates a $\sim 100 \mathrm{kHz}$ upper limit for the repetition rate of subsequent measurements. When a purged sample chamber is used, this upper limit is in the order of the minimal required interval for a gas exchange in the plasma region.

The most commonly used excitation sources in LIBS are Nd:YAG lasers operating at different harmonics. Both combine high pulse energies with repetition rates of typically around $10 \mathrm{~Hz}$. These lasers, however, have extremely low duty cycles, of the order of $10^{-7}$ taking into account their short pulse durations of $\sim 10 \mathrm{~ns}$. To increase the duty cycle and, correspondingly, the probing efficiency per given time, low power/high repetition rate lasers can alternatively be used. Several such systems have recently been introduced, e.g., diode pumped $\mathrm{Nd}: \mathrm{YVO}_{4}$ microchip lasers and fiber lasers. These lasers counterbalance the significantly lower pulse energies by their higher repetition rates of typically several (up to hundreds) $\mathrm{kHz}$. At comparable temporal pulse widths this corresponds to duty cycles of $10^{-5}$ to $10^{-3}$, i.e., 2-4 orders of magnitude higher than that of the conventional lasers. Depending on the chosen detector, the increase in observable number of plasmas per unit time can result in better counting statistics or shorter duration of individual experiments.

The dispersive systems used in LIBS are usually monochromators or spectrometers; the latter are preferred because of their multielemental capability. Typically three 
complementary types of spectrometers are used: (a) PaschenRunge spectrometers with individual photomultiplier tubes for each detected spectral line, (b) small portable CzernyTurner spectrometers with linear charged coupled device (CCD) detectors, and (c) high resolution echelle spectrometers with 2D CCD or intensified CCD, i.e., ICCD detectors. Among these types, Paschen-Runge spectrometers have some intrinsic benefits. ${ }^{1}$ They can be built very robust with very broad spectral range. The use of individual detectors per line of interest allows for an adjustment of the sensitivity of individual photomultiplier tubes (PMTs) depending on the spectral lines, with the possibility to use the cathodes with optimized sensitivity in the UV and near-infrared (NIR) regions. Different delay and integration times can be adjusted for individual PMTs which brings a vast variability into the spectroscopic measurement. Typically, Paschen-Runge spectrometers cover a wide spectral range, however, only a set of selected wavelengths can be detected if PMTs are used. Another disadvantage that Paschen-Runge and echelle spectrometers have in common is a lower light transmittance compared to a Czerny-Turner setup.

Recently, Gravel et al. ${ }^{14}$ directly compared an echelle spectrometer and a Czerny-Turner spectrometer, with respect of the limits of detection (LODs) for trace elements in aluminum and copper alloys. They found that compact spectrometers are especially favorable in combination with high repetition rate lasers. The Czerny-Turner spectrometers are more compact and affordable; they usually have a fiber-coupled input slit and a CCD line detector that only needs several milliseconds for readout. Their spectral resolution is relatively low (typically about 5000) but suffices for most LIBS applications. ${ }^{15,16}$ However, the detector cannot be temporally gated to circumvent accumulation of the white light from early stages of the plasma evolution.

Operation of echelle spectrometers with two-dimensional ICCDs or CCDs on the other hand is relatively slow (the readout takes a fraction of a second) but have spectral resolutions of up to 100000 . Using a CCD with a mechanical chopper or gated ICCD allows for temporal discrimination of the plasma radiation. In ICCDs the gating is realized by applying a rectangular high voltage (HV) pulse to the intensifying microchannel plate (MCP). Apart from the high cost and large size of these systems, the repetitive gating is usually limited to a few $\mathrm{kHz}$. This is due to the inertia of HV switches whose speed is limited by high capacitance of the MCP. Another drawback of pixel-structured detectors is their susceptibility to blurring the detected images by intensity spilling between neighboring pixels. ${ }^{17}$ The accompanying loss in spectral resolution is in strong contrast to the original resolution of the systems.

Apart of the better duty cycle, the high repetition LIBS technique benefits from good spatial profiles of microchip lasers as well as from their very low pulse-to-pulse fluctuations in terms of laser power. The lower pulse energy of the microchip lasers has to be well focused to reach the breakdown threshold of sampled materials and produce radiant micro-plasmas. Due to the lower pulse energy microchip laser-induced plasmas have shorter life times and significantly lower background compared to laser- induced plasmas produced by commonly used high-energy lasers. $^{18}$

Freedman et al. ${ }^{19}$ highlighted the benefits of the use of a microchip laser and a non-gated, CCD array detector in LIBS measurements of aluminum alloys. Nonetheless, the obtained limits of detection (LOD) are roughly two orders of magnitude higher compared to temporally gated detection. Cristoforetti et al. ${ }^{20}$ measured aluminum alloy standards with various trace elements employing a high repetition rate microchip laser and two detection systems: an Echelle spectrometer coupled to an ICCD and a Czerny-Turner spectrometer coupled to a CCD. Both systems yielded LODs in the ppm range with slightly better LODs for the Czerny-Turner-CCD system. Lopez-Moreno et al. ${ }^{15}$ used microchip LIBS for analysis of low-alloy steel standards and detected various trace elements in the ppm level. Hoehse et al. ${ }^{21}$ directly compared the analytical performance of a low repetition rate but high energy Nd:YAG and a high repetition rate but low energy microchip lasers using an Echelle-CCD detection. With both systems comparable LODs at the low ppm level could be achieved for $\mathrm{Cu}, \mathrm{Cr}$, and $\mathrm{Ni}$ in iron samples with a slightly better performance of the microchip laser spectrometer.

For field applications where LIBS systems with a minimal footprint are desired, a particularly beneficial combination consists of a small diode pumped solid state (DPSS) laser operating at a high repetition rate and a portable spectrometer with a non-intensified CCD detector. Previously, such setups have been utilized by several groups ${ }^{18-20}$ to quantify trace elements in aluminum and steel alloys with promising detection limits of hundreds of ppm. In these works, a direct comparison of non-intensified CCD spectrometers with high resolution ICCD spectrometers showed that the additional dark current noise from the microchannel plates in the ICCDs led to an increase (i.e., worsening) of the LODs. However, in the experiments with non-intensified CCD detectors no means were employed to block the initial plasma continuum. Such detectors would obviously perform even better if the continuum would be temporally eliminated by an inexpensive, portable, and robust gating device.

A desirable improvement of the above mentioned setups would, thus, be the introduction of an optical switch in front of the spectrometer to mask the undesired emission during or directly after the plasma formation. There are several demands to the switching device other than its size, cost, and robustness. A typical delay time between the laser pulse and the ideal detection window is several hundreds of nanoseconds; hence the switch needs fast opening time of $\mathrm{t}_{0} \sim 100 \mathrm{~ns}$. Furthermore, a typical plasma lifetime is between $5 \mu \mathrm{s}$ and $20 \mu$ s resulting in a maximal optical shutter frequency $\mathrm{f}_{\text {shutter }}$ $\sim 200 \mathrm{kHz}$.

Sakka et al. ${ }^{22}$ described a new LIBS detection scheme in which the first order diffraction of an acousto-optical modulator (AOM) was used to temporally control the displacement of the beforehand collimated plasma light. The light diffracted into the first order was guided into the spectrometer at a prescribed delay time with respect to the laser pulse. In that way the AOM acted as an optical switch fulfilling the requirements listed above. For the "conventional" $10 \mathrm{~Hz}$ LIBS experiment, the authors demonstrated a dramatic improvement in quality 
(a)

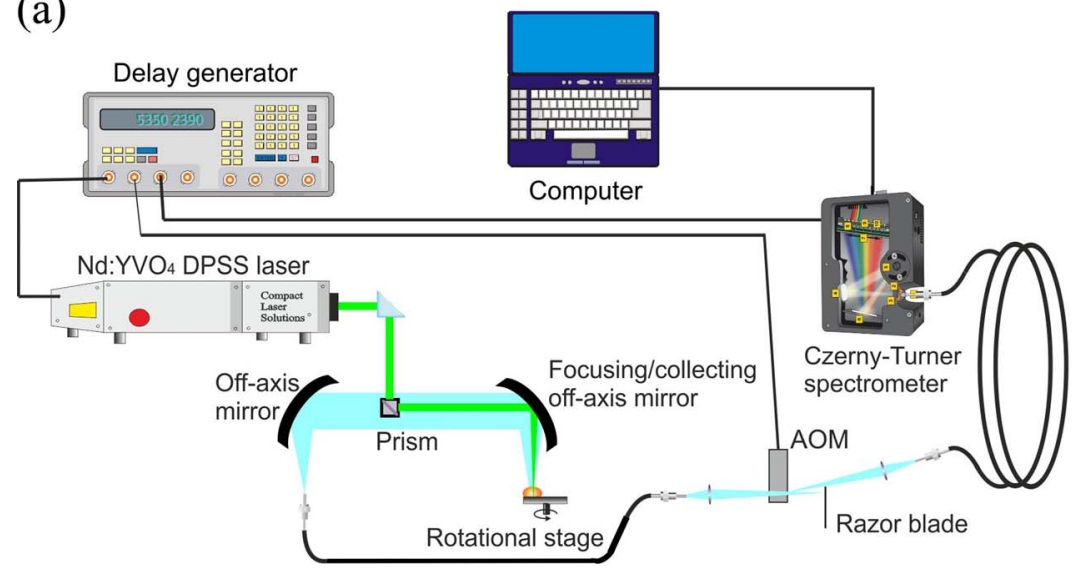

(b)

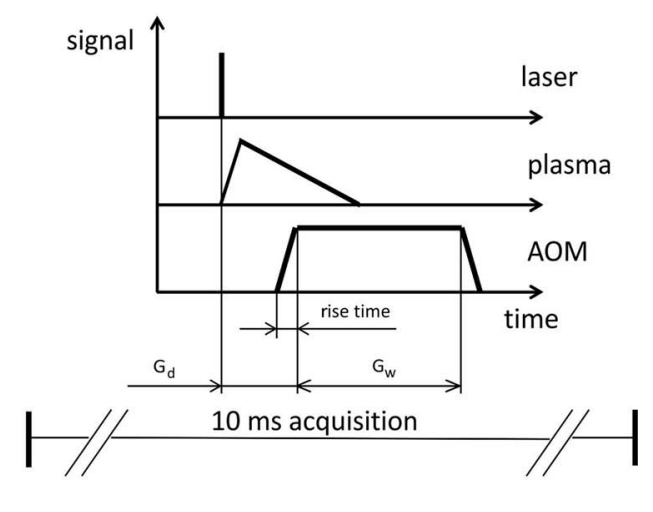

FIG. 1. (a) Schematic diagram of the experimental setup; (b) time diagram of the individual events.

of the obtained spectra using the AOM as compared to the non-gated detection. Yet, until now the main advantage of this new approach, namely, its compatibility with high repetition rate experiments, remained unexplored.

The present contribution fills this gap. It introduces a LIBS setup which includes a high repetition rate DPSS laser and a miniature Czerny-Turner spectrometer with a fast linear $\mathrm{CCD}$ detector gated by an AOM. Because of the overall lower emission of DPSS laser-induced plasmas and additional light losses in the AOM based gating process, the Czerny-Turner setup with its high light transmission appear as ideal candidates for this approach. The original advantages of the small and robust high speed instrument are now complemented by the temporal gating of the detected signal.

\section{EXPERIMENTAL}

A schematic view of the experimental setup is depicted in Fig. 1(a). A passively Q-switched $\mathrm{Nd}: \mathrm{YVO}_{4}$ DPSS laser (Blade IR25, Compact Laser Solutions GmbH, DE) operates at its fundamental wavelength of $\lambda=1064 \mathrm{~nm}$ at discrete repetition rates of 1,2,5,10,20, and $50 \mathrm{kHz}$. The pulse energies and widths of the individual laser pulses are 1.44, 1.44, 1.26, $0.96,0.58$, and $0.35 \mathrm{~mJ} /$ pulse and $18,18,18,24,36$, and $70 \mathrm{~ns}$ full width at half maximum (FWHM), respectively. The laser beam is focused onto the sample from above by an off-axis parabolic mirror (102 $\mathrm{mm}$ focal length, $38 \mathrm{~mm}$ in diameter) to create the repetition rate-dependent irradiances of 710,710, $620,350,140$, and $45 \mathrm{MW} \mathrm{cm}^{-2}$ and ignite transient plasmas. The emitted light is collimated with the same parabolic mirror and then focused with another off-axis parabolic mirror into an aperture of an optical fiber $(600 \mu \mathrm{m})$ with a second identical off-axis parabolic mirror. The light from the fiber is refocused by a $20 \mathrm{~mm}$ focal lens to a focal plane located behind the active medium of the AOM (1206C-2-1002, Isomet, USA). The optic medium of the $\mathrm{AOM}$ is $\mathrm{TeO}_{2}$, the standard transmission range is $340-420 \mathrm{~nm}$, and the mean displacement is $5.8 \mathrm{mrad}$ for the central wavelength of $\lambda=390 \mathrm{~nm}$. A spatial separation of zero and first order diffraction occurs over the entire spectral region. When the output signal of the AOM-driver (533C-L, Isomet, US) is applied to the piezo- electric transducer in the modulator, a Bragg grating is induced in the AOM crystal; the light is displaced by diffraction, and spectrally dispersed.

A razor blade was carefully positioned in front of the second focusing lens to block the zero-order light from entering the collection optics. The first order spectrally diffracted light was re-collimated (to minimize the spectral dispersion by the AOM and use it merely as an optical switch) by a $75 \mathrm{~mm}$ planoconvex lens and refocused by a $50 \mathrm{~mm}$ planoconvex lens into a second fiber that was connected to the spectrometer (USB 4000, Ocean Optics, USA). This miniaturized spectrometer is in the asymmetrical crossed Czerny-Turner configuration with $\mathrm{f} / 4$ and 1800 grooves $/ \mathrm{mm}$ grating, set to a spectral range of $200-430 \mathrm{~nm}$, with the spectral resolution $\Delta \lambda=0.4 \mathrm{~nm}$ at $315 \mathrm{~nm}$. A pulse generator (DG535, Stanford Research Systems, USA) was used for synchronization of the individual events. This spectrometer is provided with a linear CCD-array detector (with 3648 pixels of $8 \times 20 \mu \mathrm{m}$ dimension). The data readout and processing was done with a standard computer. The diffraction efficiency of the AOM was determined experimentally as the ratio of spectrally integrated intensity diffracted into the first order and that of the non-diffracted light. This value was $20 \%$. This efficiency is not as high as could be desired, however, LIBS is not a lowlight application. A desirable increase in detected light can be readily obtained by integrating the collected plasma emission over a larger solid angle or/and larger number of laser pulses. The rise time of the AOM, i.e., the time needed to build up the transient grating in the active medium, was experimentally determined to $t_{\mathrm{rt}}=70 \mathrm{~ns}$.

The time sequence of the individual events is depicted in Fig. 1(b). At a prescribed time after the laser pulse, a $24 \mathrm{~V}$ boxcar pulse with $10 \mu$ s width $(\mathrm{Gw})$ (plus twice the empirically determined rise time $t_{r t}$ ) was sent to the AOM. After the time $t_{r t}$, the AOM had established a stable grating and diffracted a constant fraction of the plasma light towards the detector.

The delay time $(\mathrm{Gd})$ was experimentally determined in order to take into account the rise time of the AOM as well as the internal delay between the Q-switch pulse and the actual firing of the laser. This cycle of the plasma ignition and 
gated detection was then repeated at different repetition rates and different delays of the detection window Gw. For a direct comparison of experiments with different repetition rates, the light was accumulated using a constant exposure time of the CCD-chip. To demonstrate the capability of the setup for fast spectrochemical analysis, the integration time was set to a short value of $10 \mathrm{~ms}$ that corresponds to 100 readouts per second and 100 laser pulses per readout (for $10 \mathrm{kHz}$ repetition rate experiments). This setting allows for fast detection of elemental composition $(100 \mathrm{~Hz})$ while in each individual analysis the signal-to-noise statistics benefit from 100 individual consecutive plasma events. For LIBS measurements without temporal gating, the plasma radiation was collected with the same collection optics and introduced into the working area of the AOM. For detection of ungated signal, however, the Bragg grid was constantly present inside the AOM so the entire plasma light including the continuous radiation and bremsstrahlung was diffracted with no temporal gating. Only radiation dispersed into the 1st order of diffraction was accumulated on the CCD line detector. This setting was used to obtain non-gated spectra for later comparison with gated spectra. It is worth mentioning that the AOM only deflected $\sim 20 \%$ of the incident beam. Thus, $\sim 80 \%$ of the signal detected in the ungated experiments was merely lost in the Bragg grating diffraction and never reached the detector. Signal statistic dictates that this inefficiency leads to a decrease in signal to noise of factor $\frac{\sqrt{1}}{\sqrt{0.2}}=2.24$. Even though the factor 5 in light collection efficiency can readily be compensated in most setups by using more efficient collection optics, this signal loss is one intrinsic drawback of the proposed gating. The described effect can, however, be minimized by the use of more efficient AOM materials.

At each operational mode, three individual spectra were recorded and mean values of line intensities were used to optimize the setup. The spectral range of the spectrometer exceeds the transmittance range of the AOM crystal material. Therefore, beyond the overlapping region, the recorded data contained only dark current and stray light but no plasma emission. The aluminum standard 309 with low Mg content was used as a blank sample; a linear fit to the baseline of this sample spectrum in the region of 380-385 nm served for the background correction of plasma spectra. For data analysis, standard software was employed. Every spectrum was smoothed with a 3 point Savitzky-Golay algorithm and all peaks were fitted with a Gaussian profile. The authors are aware of the Voigt beam profile of the spectral lines. However, as a good approximation Gaussian beam profiles were used throughout. As a further simplification, the heights of the peaks were taken as line intensities, neglecting changes in the peak widths.

\section{RESULTS AND DISCUSSION}

The feasibility study of the proposed approach started with optimization of the temporal and spatial parameters of the conducted experiment. In a subsequent study to estimate the analytical applicability, the LOD for $\mathrm{Mg}$ in $\mathrm{Al}$ alloy standard reference materials (SRM) (BAM, Germany) was obtained.

\section{A. Optimization of experimental parameters}

To provide the correct timing for experiments with different repetition rates, the internal delay between the trigger of the Q-switch and the actual emission of the laser pulse was determined for each repetition rate individually using a fast photodiode (DET10A, Thorlabs). For the repetition rates between $1 \mathrm{kHz}$ and $50 \mathrm{kHz}$, the delays varied between $450 \mathrm{~ns}$ and $730 \mathrm{~ns}$.

Consecutively, the rise time $t_{r t}$ of the Bragg grid inside the AOM was experimentally determined by probing the diffracted part of a continuous light source by the photodiode. The acoustic wave is created on one side of the AOM and is propagated to the working area within a finite time interval. This causes the delay in establishing the stable grating inside the AOM and determines the rise time during which the fraction of the diffracted light increases. The internal delays of both devices, DPSS laser and AOM, were taken into account in all subsequent measurements.

The geometrical design of the setup, i.e., the mutual arrangement of optical elements, was optimized by maximizing the intensity of the $\mathrm{Al}$ (I) line at $\lambda=396.1 \mathrm{~nm}$ in the aluminum alloy 312 (see Table I). Even though this line could be self-absorbed, a maximum signal on the detector still corresponded to the most efficient collection and coupling of the emitted light. Temporal optimization of AOM gating was conducted at a fixed repetition rate of the DPSS laser $(10 \mathrm{kHz})$ while the detection window at a fixed width of $10 \mu$ s was altered by shifting the AOM gating pulse with respect to the laser pulse from $-400 \mathrm{~ns}$ to $600 \mathrm{~ns}$.

The results are depicted in Fig. 2(a) and several interesting features can be identified from these spectra. From $100 \mathrm{~ns}$ on, the overall intensity of the $\mathrm{Al}$ (I) doublet at $\lambda=$ 394.4 and $396.1 \mathrm{~nm}$ decreases with further increase of the gate delay $(\mathrm{Gd})$. This decrease can be explained by detection of less plasma continuum and by gradual plasma cooling. There is another broad spectral feature around 358-359 nm that appears only in the early plasma and completely vanishes by $300 \mathrm{~ns}$ after the formation of the plume. This feature is attributed to a strong $\mathrm{Al}$ (II) triplet at $358.6 \mathrm{~nm}, 358.7 \mathrm{~nm}$, and $358.7 \mathrm{~nm}$; the upper state of this triplet at $15.3 \mathrm{eV}$ can only be significantly populated in early and hot plasmas. ${ }^{20}$ Even though the overall intensity of the Al (I) doublet decreases

TABLE I. Elemental composition of aluminum standards from Federal Institute for Materials Research and Testing (BAM, Germany).

\begin{tabular}{lccccccccc}
\hline \hline \multirow{2}{*}{$\begin{array}{l}\text { Al alloy } \\
\text { no. }\end{array}$} & $\mathrm{Al}$ & $\mathrm{Cr}$ & $\mathrm{Fe}$ & $\mathrm{Mg}$ & $\mathrm{Mn}$ & $\mathrm{Si}$ & $\mathrm{Zn}$ & Remaining \\
\cline { 2 - 10 } & & & & & & \\
307 & 93.72 & 0.16 & 0.41 & 4.58 & 0.70 & 0.16 & 0.06 & 0.21 \\
308 & 90.21 & 0.20 & 0.16 & 2.29 & 0.03 & 0.07 & 5.67 & 1.36 \\
309 & 88.02 & $\ldots$ & 0.09 & $6.8 \times 10^{-5}$ & 0.05 & 11.76 & $\ldots$ & 0.08 \\
310 & 98.81 & $\ldots$ & 0.07 & 0.99 & $\ldots$ & 0.08 & 0.01 & 0.04 \\
311 & 91.86 & 0.10 & 0.31 & 1.57 & 0.69 & 0.20 & 0.20 & 5.06 \\
312 & 98.79 & 0.03 & 0.19 & 0.41 & 0.04 & 0.42 & 0.03 & 0.11 \\
313 & 94.73 & 0.12 & 0.39 & 3.40 & 0.50 & 0.36 & 0.16 & 0.34 \\
314 & 82.98 & 0.05 & 0.76 & 0.18 & 0.40 & 11.49 & 1.20 & 2.94 \\
\hline \hline
\end{tabular}



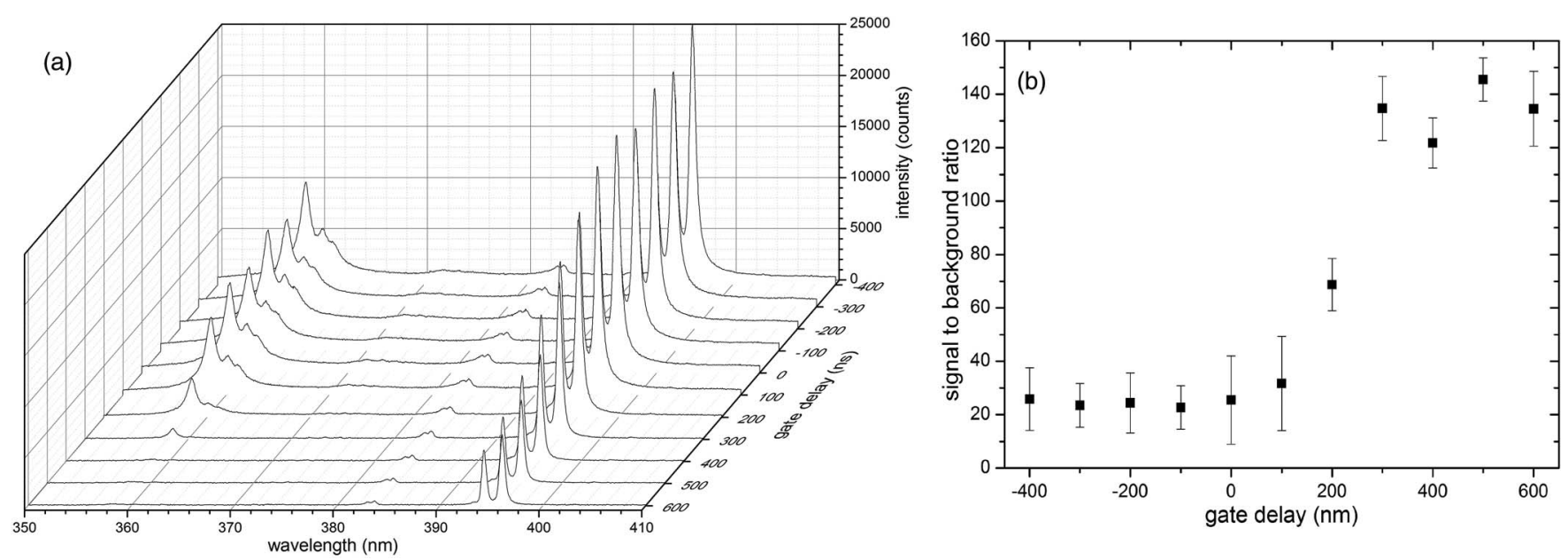

FIG. 2. (a) AOM-gated LIBS spectra of Al alloy for different delay times. (The delay time corresponds to the real temporal positions. The internal delays in the $\mathrm{AOM}$ and laser are already considered in these numbers.) (b) signal-to-background ratio of the $\mathrm{Al} \mathrm{I} 396.15 \mathrm{~nm}$ line for different delay times.

towards later detection window, an increase in the slope of the profiles, i.e., a better contrast, can be observed.

One sees from Fig. 2(a) that spectral background between the spectral bands decreases as well with increasing the delay time. The signal-to-background ratio (SBR) for the $\mathrm{Al}$ (I) line at $396.1 \mathrm{~nm}$ is plotted in Fig. 2(b) as a function of the delay time. A 7-fold increase in SBR due to the temporal gating can be observed starting from 200 ns. The SBR starts rising at around $\mathrm{Gd}=100 \mathrm{~ns}$, then reaches the maximum at $300 \mathrm{~ns}$ and saturates. Therefore, all subsequent measurements were conducted with the AOM delay time fixed at $\mathrm{Gd}=300 \mathrm{~ns}$.

Subsequently, the effect of the laser repetition rate on the absolute intensity of detected emission signal was tested.
Fig. 3 shows the $\mathrm{Al}$ alloy spectra recorded at a fixed gate delay of $\mathrm{Gd}=300 \mathrm{~ns}$ for different repetition rates ranging from $1 \mathrm{kHz}$ to $50 \mathrm{kHz}$. For the rates between 1 and $10 \mathrm{kHz}$ an almost linear increase in line intensities can be observed, while at the rates higher than $20 \mathrm{kHz}$ the emission signal significantly drops. This is because the DPSS laser has an almost constant pulse power only at rates below $5 \mathrm{kHz}$. At higher repetition rates the power of the pumping diodes does not suffice to maintain a constant population inversion within the lasing media; this results in the decrease of output pulse energy, and, hence, of irradiance on the sample surface. Another reason for the decrease in irradiance is the longer pulse durations at higher repetition rates. Even though the gate width of

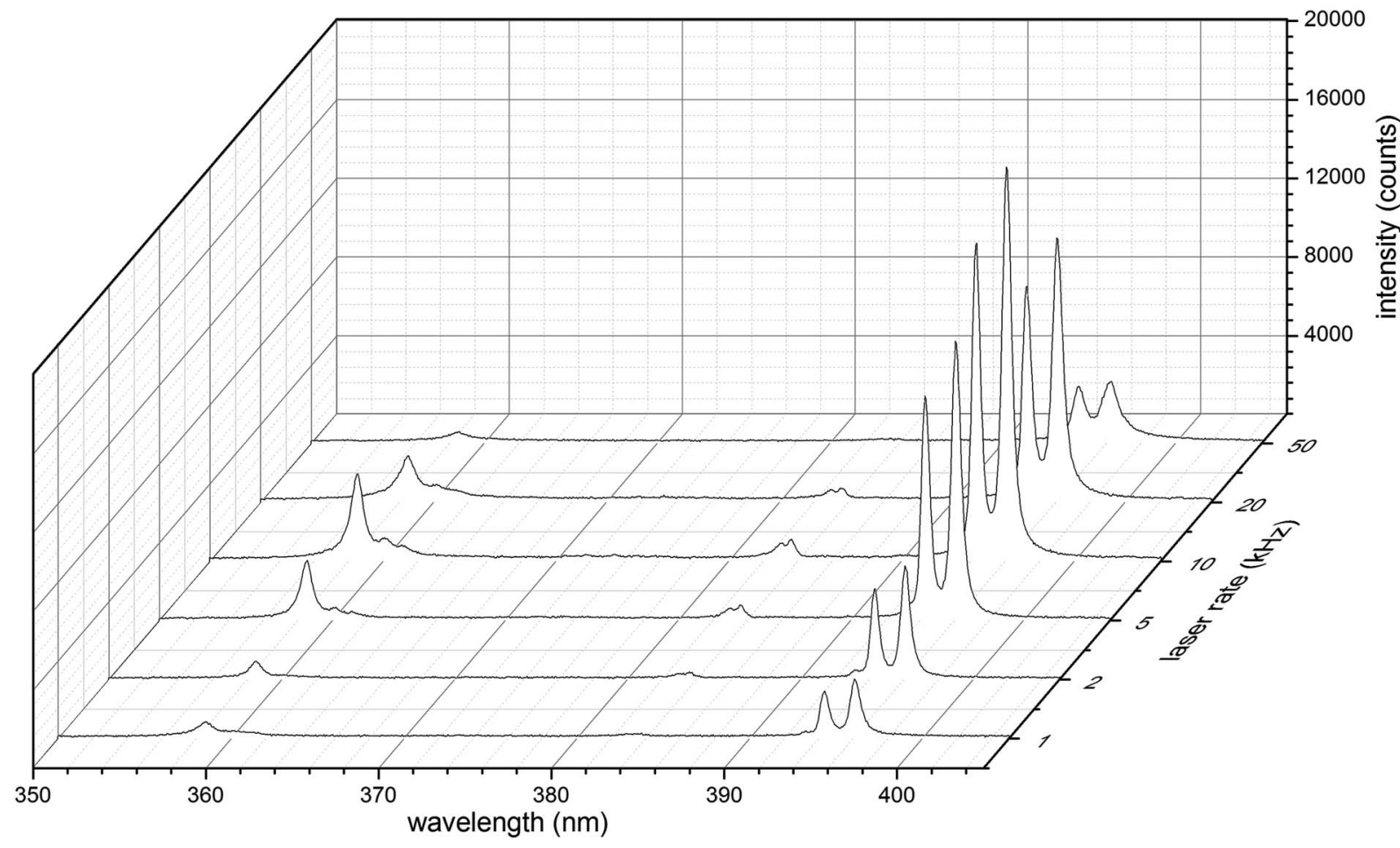

FIG. 3. LIBS spectra of a typical $\mathrm{Al}$ alloy for different experimental repetition rates. 
$\mathrm{Gw}=10 \mu \mathrm{s}$ would allow for repetition rates of up to $100 \mathrm{kHz}$, all subsequent measurements were conducted at $10 \mathrm{kHz}$.

As discussed in the LIBS literature, multiple laser pulses impinging on the same sample spot affect the signal intensity. A positive effect is the so-called laser cleaning where subsequent laser pulses ablate a fresh surface after a corrosion layer has been removed. However, after a certain number of laser pulses, the laser drills a hole through the surface and light from later pulses is trapped inside the crater or blocked by the crater's rim. In the case of the angular or side-view observation geometries this light does not reach the detector. The effect is more pronounced at larger numbers of laser pulses and higher fluencies. In experiments with high repetition rate lasers, the laser hits the target repeatedly within a short time interval $(100 \mu \mathrm{s}$ at $10 \mathrm{kHz})$. After the impact of each laser pulse on a metal target the interaction spot partially melts and a LIP is produced. At repetition rates of above $1 \mathrm{kHz}$ it is reasonable to expect that the melted phase does not resolidify prior to the subsequent laser pulse and thus hampers the plasma formation since a melted metallic phase exhibits a higher optical breakdown threshold compared to their solid counterparts. ${ }^{15,18}$ The higher the repetition rate, the less time the melt phase has to cool down and solidify. The heat conduction within the sample is not able to transport a sufficient amount of the absorbed energy for solidification of the interaction region. The same effect could be observed in an earlier study using the same laser system at comparable repetition rates. ${ }^{21}$ Gornushkin et al. ${ }^{15}$ recommended to move the target during the analysis to avoid the occurrence of a melt phase, which does not have time to solidify, in the interaction region. This phenomenon was observed when two consequent laser pulses were delivered with the delay $\sim 0.2 \mathrm{~ms}$, corresponding to $5 \mathrm{kHz}$ laser repetition rate.

As mentioned earlier, in typical LIBS experiments either a low number of high power laser pulses at repetition rates of several $\mathrm{Hz}$ or a vast number of low power laser pulses at rates of several $\mathrm{kHz}$ are applied to collect an analytical signal. In the present experiment the untypical sequence of only 100 quickly succeeding laser pulses with low fluence was used. For this experiment, the influence of the sample movement on the analytical signal was tested. For this, the signal obtained from a constantly moving sample (achieved by mounting the sample onto the chuck of a handheld drill operated at a rotational speed of 33000 rounds/min) was directly compared to the signal recorded with simple manual movement of a handheld sample. A thorough comparison, however, showed only negligible difference in the spectral intensities. Hence, for the relatively small number of 100 subsequent laser pulses, manual movement of the samples was applied throughout.

\section{B. Al samples}

To emphasize the benefits arising from the temporal gating with the AOM, Fig. 4 comparatively shows the two spectra recorded with (bold) and without gating. For easier visual comparison, the spectra were normalized to the highest intensity of the aluminum line at $396.1 \mathrm{~nm}$. Apart from the significantly increased SBR, two other features become vis-

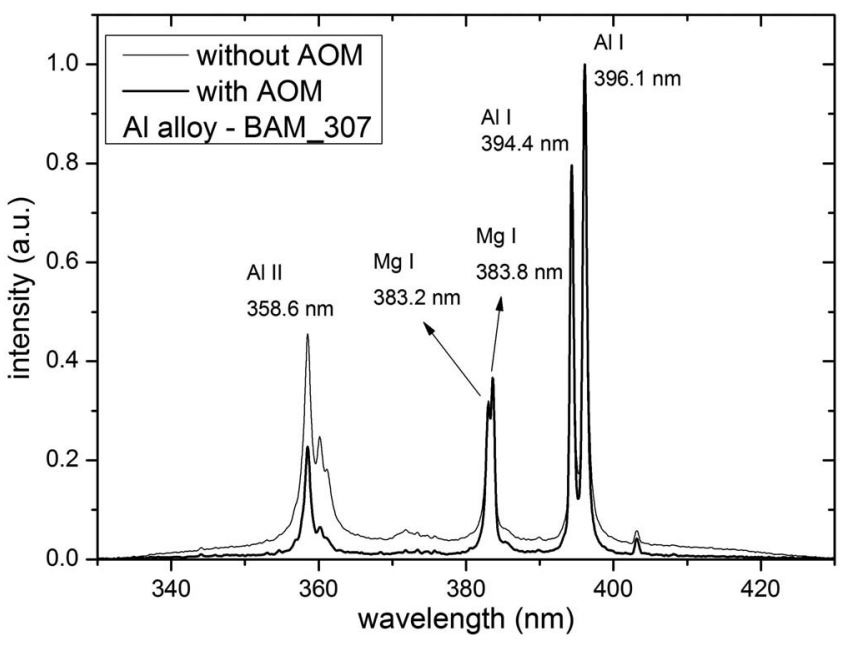

FIG. 4. Direct comparison of normalized LIBS spectra of Al alloy sample with (bold) and without temporal gating with AOM.

ible from Fig. 4. The reduced background appears only in the region of transparency of the AOM medium, from 340 to $420 \mathrm{~nm}$. The spectra show a strongly broadened Al (II) triplet at $358.6-358.7 \mathrm{~nm}$. The reduction of the ionic line intensity is evident for the measurement utilizing AOM temporal gating set at $300 \mathrm{~ns}$. This effect agrees with our previous observation that the contribution of ionic lines is mostly important at early stages ( $<300 \mathrm{~ns}$ ) of the plasma evolution.

To estimate the analytical applicability of the suggested approach, aluminum alloy standard reference materials (BAM, Germany) whose elemental composition is listed in Table I, were measured. Special emphasis was put onto the observation of trace elements, especially magnesium, see Fig. 5(a). The two magnesium lines, $\mathrm{Mg}$ (I) $383.2 \mathrm{~nm}$ and $\mathrm{Mg}$ (I) $383.8 \mathrm{~nm}$, strongly overlap due to the low spectral resolution of the Czerny-Turner spectrometer. The amount of $\mathrm{Mg}$ in the sample 309 is lower than LODs in proposed system. In Fig. 5(a) the sample 309 is depicted however no Mg peaks can be observed.

The calibration curves for magnesium in aluminum alloys obtained with and without the AOM temporal gating are shown in Fig. 5(b). The analytical signal was corrected by the background intensity employing a blank sample measurement (aluminum standard no. 309 containing merely $6.8 \times$ $10^{-5}$ wt.\% of magnesium was considered as the blank sample). In the complete analysis the height of the Mg I peak at $383.8 \mathrm{~nm}$ served as the analytical signal. The LODs were computed employing to the $3 \sigma$-IUPAC criterion. ${ }^{23,24}$ Accordingly, the LODs were computed by the equation $L O D=\frac{3 \sigma}{s}$, where $\sigma$ is the standard deviation of the background at the wavelength in a blank sample and $s$ is the slope of the calibration curve, respectively. For this quantification analysis the raw data served as input, no further normalization or internal standardization was used throughout.

The improvement of the LODs due to the use of the AOM is already obvious from Fig. 4. The lowest detectable concentration of $\mathrm{Mg}$ was 0.13 wt.\% obtained with the setup with the DPSS laser and AOM and with accumulation of 300 laser pulses per sample. The LOD of $0.625 \mathrm{wt} . \%$ for the LIBS setup 

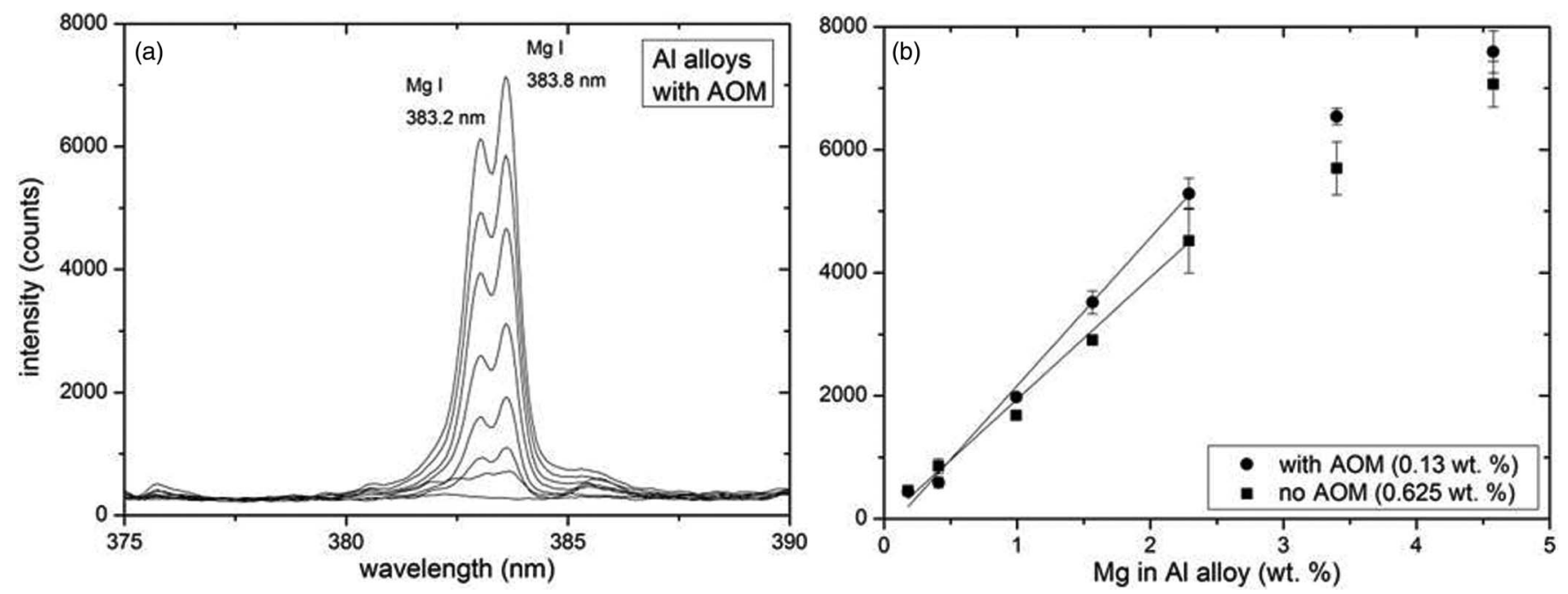

FIG. 5. (a) LIBS spectra of different aluminum standards with the emphasis on magnesium. (b) Calibration curve for Mg in aluminum standards.

without the AOM gating are significantly higher than that in the case of the gated detection. Even though the gating mechanism sacrifices roughly $80 \%$ of the emitted light before it reaches the detector, the observed fivefold improvement in LODs outweighs the improvement that can be estimated from collecting the entire light entering the AOM (factor 2.24, see above). Future improvements can be expected from AOMs built from different active media with a more efficient diffraction. This result strengthens the importance of temporal gating of laser-induced plasma emission and usability of the AOM for this application. Compared to results from the literature, ${ }^{20}$ the obtained LODs employing the AOM-gated setup appear to be large. However, the previous experiments were not aiming towards fast detection and a vast portion of the improvement in LODs are likely to stem from the significantly lower amount of accumulations employing the AOM-gated setup (100) compared to the number of accumulations used in Ref. 20 (160 000). Furthermore, in Ref. 1 (p. 451) a limit of detection is reported for the same $\mathrm{Mg}$ (I) line $(383.8 \mathrm{~nm})$ in $\mathrm{Al}$ matrix of $0.057 \%$. Those LODs were achieved with so-called FML system (Flexible Measuring System based on LIBS) with a sequence of 100 laser pulses. The FML system consists of compact Nd:YAG laser with pulse energy of $50 \mathrm{~mJ}$ and a Paschen-Runge spectrometer equipped with three CCDs covering the spectral range from 278 to $560 \mathrm{~nm}$.

Though the results presented here seem to be unsatisfactory compared to the references, the aim of our investigation was a mere proof of principle. The lower detection limits in Ref. 1 most likely result from the higher irradiance of individual laser pulses and the better spectral resolving power of the used spectrometer. In future experiments the obtained LODs can be improved using AOMs with better throughputs, bringing more efficient light transmittance.

In summary, an AOM can be used for temporal gating of light emitted from transient laser-induced plasmas. In LIBS applications, this gating can be used to significantly decrease the limits of detection. Since AOMs are small and robust components with low power consumption and no mechanical parts, they seem ideal for the future development of compact high-rate LIBS devices. On the other hand, AOMs limit the wavelength range and as well the intensity of transmitted plasma radiation. Optical system for collecting the plasma radiation is of high importance. AOMs essentially decrease the intensity of temporally gated radiation, which is diffracted on Bragg grid within the active medium of AOM. Therefore, the choice of AOMs has to be adapted to the case of the study.

\section{CONCLUSIONS}

The use of acousto-optical modulators as fast optical shutters allows convenient time gating in LIBS experiments with high repetition rate lasers. Due to the low price, small size, and the possibility of fiber coupling of these optical shutters to spectrometers, almost any experimental setup can be easily equipped with an AOM gating unit. The small and compact LIBS setup with the AOM temporal gating was successfully utilized for the detection of traces of $\mathrm{Mg}$ in aluminum alloy standard reference materials. Moreover, improved signalto-background ratio (SBR) for the lines of $\mathrm{Mn}, \mathrm{Si}$, and $\mathrm{Cr}$ in the analyzed aluminum alloys was observed, not shown in the article. The achieved LODs for Mg employing AOM-gated setup were $\sim 0.13 \mathrm{wt} . \%$. These results show improvement in comparison with non-gated detection $(0.625 \mathrm{wt} . \%)$. The relatively poor LODs in general result from the short integration time of the individual experiments in combination with the high-rate laser source when a temporal detection cannot be provided utilizing conventional ICCDs. Additionally, none of the state of the art normalization procedures to the intensity of a matrix elemental line has been applied. However, even at an integration time of only $10 \mathrm{~ms}$, the described experimental LIBS setup could satisfy the needs of semi-quantitative spectrochemical analysis of up to 100 spots/s. Additionally, the absence of any moving parts makes the AOM-shutter an ideal gating device in harsh environments.

\section{ACKNOWLEDGMENTS}

Authors gratefully acknowledge funding from the program Zentrale Innovationsprogramm Mittelstand of the 
German Federal Ministry of Economics and Technology. The work of I.G. and U.P. is supported in part by the DFG-NSF Grant No. GO 1848/1-1 (Germany).

${ }^{1}$ R. Noll, Laser-Induced Breakdown Spectroscopy: Fundamentals and Applications (Springer-Verlag, Berlin, 2012).

${ }^{2}$ A. W. Miziolek, V. Palleschi, and I. Schechter, Laser-Induced Breakdown Spectroscopy (LIBS): Fundamentals and Applications (Cambridge University Press, Cambridge, 2006).

${ }^{3}$ D. A. Rusak, B. C. Castle, B. W. Smith, and J. D. Winefordner, Crit. Rev. Anal. Chem. 27, 257-290 (1997).

${ }^{4}$ R. H. Scott and A. Strasheim, Spectrochim. Acta, Part B 25, 311-332 (1970).

${ }^{5}$ D. A. Cremers, L. J. Radziemski, and T. R. Loree, Appl. Spectrosc. 38, 721-729 (1984)

${ }^{6}$ M. Sabsabi and P. Cielo, Appl. Spectros. 49, 499-507 (1995).

${ }^{7}$ P. Pořízka, D. Prochazka, Z. Pilát, L. Krajcarová, J. Kaiser, R. Malina, J. Novotný, P. Zemánek, J. Ježek, M. Šerý, S. Bernatová, V. Krzyžánek, K. Dobranská, K. Novotný, M. Trtílek, and O. Samek, Spectrochim. Acta, Part B 74-75, 169-176 (2012).

${ }^{8}$ R. Knopp, F. J. Scherbaum, and J. J. Kim, Fresenius' J. Anal. Chem. 355, 16-20 (1996).

${ }^{9}$ S. Nakamura, Y. Ito, K. Sone, H. Higara, and K. Kaneko, Anal. Chem. 68, 2981-2986 (1996).

${ }^{10}$ C. Haisch, R. Niessner, O. I. Matveev, U. Panne, and N. Omenetto, Fresenius' J. Anal. Chem. 356, 21-26 (1996).

${ }^{11}$ V. Hohreiter and D. W. Hahn, Anal. Chem. 77, 1118-1124 (2005).
${ }^{12}$ D. W. Hahn and N. Omenetto, Appl. Spectrosc. 66, 347-419 (2012).

${ }^{13}$ R. Noll, C. Fricke-Begemann, M. Brunk, S. Connemann, C. Meinhardt, M. Scharun, V. Sturm, J. Makowe, and C. Gehlen, Spectrochim. Acta, Part B 93, 41-51 (2014).

${ }^{14}$ J. F. Y. Gravel, F. R. Doucet, P. Bouchard, and M. Sabsabi, J. Anal. At. Spectrom. 26, 1354-1361 (2011).

${ }^{15}$ C. Lopez-Moreno, K. Amponsah-Manager, B. W. Smith, I. B. Gornushkin, N. Omenetto, S. Palanco, J. J. Laserna, and J. D. Winefordner, J. Anal. At. Spectrom. 20, 552-556 (2005).

${ }^{16}$ B. C. Castle, A. K. Knight, K. Visser, B. W. Smith, and J. D. Winefordner, J. Anal. At. Spectrom. 13, 589-595 (1998).

${ }^{17}$ M. Mueller, I. B. Gornushkin, S. Florek, D. Mory, and U. Panne, Anal. Chem. 79, 4419-4426 (2007).

${ }^{18}$ I. B. Gornushkin, K. Amponsah-Manager, B. W. Smith, N. Omenetto, and J. D. Winefordner, Appl. Spectrosc. 58, 762-769 (2004).

${ }^{19}$ A. Freedman, F. J. Iannarilli, and J. C. Wormhoudt, Spectrochim. Acta, Part B 60, 1076-1082 (2005).

${ }^{20}$ G. Christoforetti, S. Legnaioli, V. Palleschi, A. Salvetti, E. Tognoni, P. A. Benedetti, F. Brioschi, and F. Ferrario, J. Anal. At. Spectrom. 21, 697-702 (2006).

${ }^{21}$ M. Hoehse, I. B. Gornushkin, S. Merk, and U. Panne, J. Anal. At. Spectrom. 26, 414-424 (2011).

${ }^{22}$ T. Sakka, K. Irie, K. Fukami, and Y. H. Ogata, Rev. Sci. Instrum. 82, 023112 (2011).

${ }^{23}$ P. K. Kennedy, D. X. Hammer, and B. A. Rockwell, Prog. Quantum Electron. 21, 155-248 (1997).

${ }^{24}$ J. Mocak, A. M. Bond, S. Mitchell, and G. Scollary, Pure Appl. Chem. 69, 297-328 (1997). 